\title{
Effect of PdS on Photocatalytic Hydrogen Evolution of Nanostructured CdS under Visible Light Irradiation
}

\author{
Qingyun Chen, Cheng Suo, Shu Zhang, and Yunhai Wang \\ State Key Laboratory of Multiphase Flow in Power Engineering, School of Energy and Power Engineering, Xian Jiaotong University, \\ Xian 710049, China \\ Correspondence should be addressed to Qingyun Chen; qychen@mail.xjtu.edu.cn
}

Received 12 December 2012; Accepted 12 January 2013

Academic Editor: Elias Stathatos

Copyright (C) 2013 Qingyun Chen et al. This is an open access article distributed under the Creative Commons Attribution License, which permits unrestricted use, distribution, and reproduction in any medium, provided the original work is properly cited.

To investigate the effect of PdS as a cocatalyst for photocatalytic hydrogen evolution, nanostructured PdS/CdS were prepared by an in situ coprecipitation and hydrothermal method, respectively. The as-prepared photocatalysts were characterized by transmission electron microscopy (TEM), X-ray diffraction (XRD), UV-visible absorption spectra, and photoluminescence spectra (PL). With PdS highly dispersed in the CdS nanostructures, the photoactivity was evaluated by hydrogen evolution from aqueous solution containing $\mathrm{Na}_{2} \mathrm{~S} / \mathrm{Na}_{2} \mathrm{SO}_{3}$ as sacrificial reagents under visible light irradiation. When the concentration of PdS was $1 \%$ by weight, $\mathrm{PdS} / \mathrm{CdS}$, prepared by the in situ coprecipitation, showed the highest photocatalytic activity, while that prepared by hydrothermal method showed the most stability for hydrogen evolution. The effect of highly dispersed PdS on the photoactivity was discussed.

\section{Introduction}

Hydrogen, as a kind of renewable energy, has attracted more and more attention due to the depletion of fossil fuels and the pollution caused by continuously increasing energy demands [1-3]. Photocatalytic water-splitting hydrogen evolution using semiconducting photocatalysts is one of the most ideal ways to make full use of solar energy to produce hydrogen [4]. Up to now, a lot of semiconducting photocatalysts have been reported to produce hydrogen, such as $\mathrm{TiO}_{2}$ [5], CdS [6], and $\mathrm{ZnS}$ [7]. Among them, $\mathrm{CdS}$ has been widely studied because of its excellent light absorption property in the visible region [8].

$\mathrm{CdS}$ alone demonstrates low photoactivity and bad stability for hydrogen evolution under visible light because, in its photocatalytic process, the phenomenon of photocorrosion is prone to occur [9]. Many efforts have been made to overcome the problem of photocorrosion, such as photosensitizing $\mathrm{CdS}$ with photosensitizers $[4,10]$, combining CdS with another semiconductor [11], and exploiting alternative preparation approaches to control the size and shape of CdS [12].
Loading a cocatalyst onto CdS is also a good method to improve its photoactivity and stability. A suitable cocatalyst cannot only promote the separation of photoexcited electrons and holes and suppress their recombination, but also decrease the activation potentials for $\mathrm{H}_{2}$ evolution [13]. Transition metals and their oxides (sulfides) have been widely used as cocatalysts including Pd [14-16], Pt [17, 18], $\mathrm{NiO}$ [19], $\mathrm{RuO}_{2}$ [20], and $\mathrm{MoS}_{2}$ [21]. Therefore, it is highly desirable to find suitable cocatalysts to improve the photoactivity and stability of CdS. Recently, Yan et al. reported that CdS loaded with PtPdS bicomponent cocatalyst achieved the highest quantum yield of about $93 \%$ in the presence of sacrificial reagents under visible light irradiation [22]. Therefore, it would be of great interest to investigate the effect of the cocatalyst on the photocatalytic performance of CdS.

In this paper, nanostructured PdS/CdS photocatalysts were synthesized by in situ coprecipitation and hydrothermal method, respectively. The effect of PdS on the photoactivity and stability of $\mathrm{CdS}$ was investigated for hydrogen evolution under visible light from water containing $\mathrm{Na}_{2} \mathrm{~S}$ and $\mathrm{Na}_{2} \mathrm{SO}_{3}$ as sacrificial reagents. 


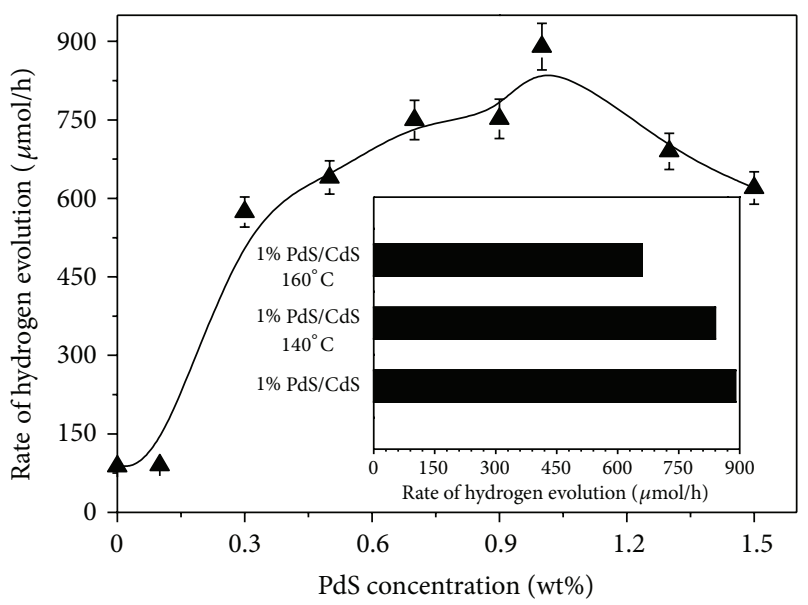

FIGURE 1: Rate of hydrogen evolution on PdS/CdS samples loaded with different amounts of PdS under visible light; (insert) rate of hydrogen evolution on PdS/CdS samples loaded with $1 \mathrm{wt} \%$ amount of PdS prepared by different hydrothermal temperatures and in situ coprecipitation.

\section{Experiments}

2.1. Synthesis of PdS/CdS. All of the chemicals were of analytical grade and used without further purification. Deionized water was used in the sample synthesis.

The nanostructured CdS particles were synthesized as follows: an aqueous solution of $0.14 \mathrm{M} \mathrm{Na}_{2} \mathrm{~S}$ was added slowly to $0.14 \mathrm{M} \mathrm{Cd}(\mathrm{OAc})_{2}$ solution under vigorous stirring at room temperature. The yellow mixture was stirred for $12 \mathrm{~h}$ and kept for additional $12 \mathrm{~h}$. The resulting yellow solid was collected by filtration and washed with deionized water several times. The wet solid was suspended in deionized water and transferred into a $100 \mathrm{~mL}$ stainless Teflon-lined autoclave. The autoclave was sealed and heated at $200^{\circ} \mathrm{C}$ for $24 \mathrm{~h}$ and then cooled down to room temperature naturally. The yellow solid was filtered and washed with water and ethanol subsequently, followed by drying at $80^{\circ} \mathrm{C}$ for $24 \mathrm{~h}$.

The loading of $\mathrm{PdS}$ on CdS producing PdS/CdS was realized by the following two methods. For the in situ coprecipitation, a $\mathrm{PdCl}_{2}$ aqueous solution was added dropwise under stirring at room temperature to a suspension of $\mathrm{CdS}$ nanoparticles ( $0.2 \mathrm{~g}$, as prepared above) dispersed in $\mathrm{Na}_{2} \mathrm{~S}$ aqueous solution just before the photocatalytic reaction. For the hydrothermal method, the mixture obtained by the in situ coprecipitation was stirred for $12 \mathrm{~h}$. The resulting precipitates were centrifuged and washed with deionized water several times. The precipitates were suspended in deionized water, transferred into a $100 \mathrm{~mL}$ stainless Teflon-lined autoclave, and heated at a certain temperature for $6 \mathrm{~h}$.

2.2. Characterization. The X-ray diffraction (XRD) patterns of catalysts were obtained from a Panalytical X'pert Pro Xray diffractometer equipped with $\mathrm{Cu} \mathrm{K} \alpha$ irradiation with the scanning step of $0.05^{\circ} / \mathrm{s}$; the operation voltage and current were $45 \mathrm{kV}$ and $40 \mathrm{~mA}$, respectively. UV-visible absorption spectra were measured by a HITACHI UV4100 instrument,

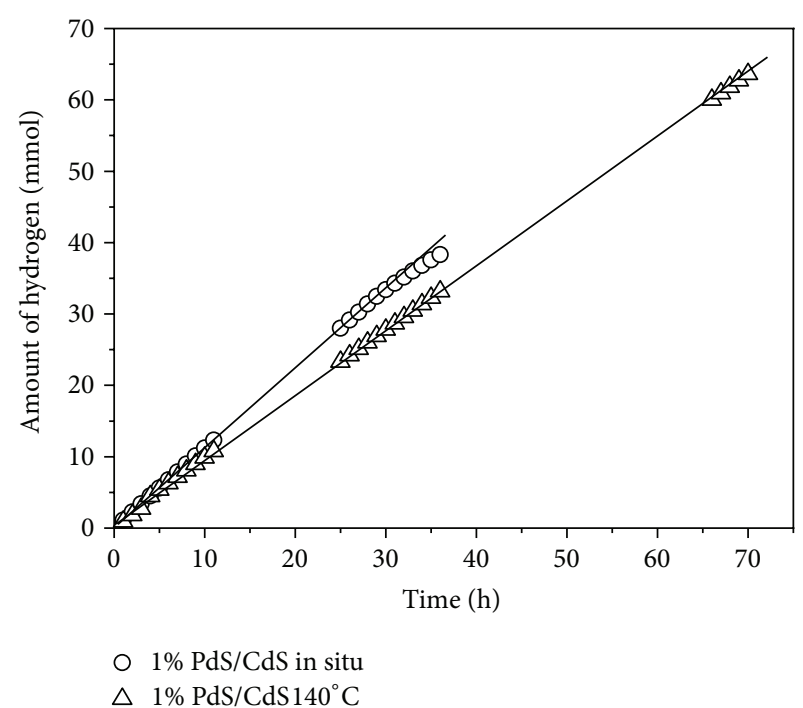

FIGURE 2: Time courses of hydrogen evolution on PdS/CdS samples loaded with $1 \mathrm{wt} \% \mathrm{PdS}$ prepared by different methods.

with the scanning range from 300 to $800 \mathrm{~nm}$. The photoluminescence spectra (PL) were measured at room temperature using a PTI QM-4 fluorescence spectrophotometer. The crystallite morphologic micrograph was observed on a high resolution transmission electron microscopy (HRTEM) JEOL JEM-3010 instrument and a field emission scanning electron microscopy (SEM) JSM-6700F (Japan).

2.3. Evaluation of Photocatalytic Activity. Photocatalytic reaction was carried out in a side-irradiation Pyrex cell. The effective irradiation area for the cell is $12.56 \mathrm{~cm}^{2}$. The powder of photocatalyst $(0.2 \mathrm{~g})$ was dispersed by a magnetic stirrer in an aqueous solution $(200 \mathrm{~mL})$ consisting of $\mathrm{Na}_{2} \mathrm{~S}(0.5 \mathrm{M})$ and $\mathrm{Na}_{2} \mathrm{SO}_{3}(0.5 \mathrm{M})$ as electron donors in the cell. The photocatalysts were irradiated with visible light through a cutoff filter $(\lambda>430 \mathrm{~nm}, T=65 \%)$ from a $300 \mathrm{~W}$ Xe lamp. The amount of $\mathrm{H}_{2}$ gas was determined by an online thermal conductivity detector (TCD) gas chromatograph ( $\mathrm{NaX}$ zeolite column, nitrogen as a carrier gas).

\section{Results and Discussion}

Figure 1 shows the rate of hydrogen evolution on PdS/CdS photocatalysts loaded with different amounts of PdS prepared by the in situ coprecipitation under visible light, together with those prepared by the hydrothermal method (insert in Figure 1). As the amount of PdS increases from 0 to $1 \%$, the hydrogen evolution rate on PdS/CdS increases markedly from $\sim 88.1 \mu \mathrm{mol} / \mathrm{h}$ to $\sim 890.3 \mu \mathrm{mol} / \mathrm{h}$, about 10 times higher. However, when the amount of PdS increases to $1.3 \%$, the photocatalytic activity decreases slightly. The surplus PdS can work as an optical filter to shield incident light and hence suppress further enhancement of photocatalytic activity for hydrogen evolution. For comparing, the insert in Figure 1 shows the rate of hydrogen evolution on the PdS/CdS photocatalyst loaded with $1 \mathrm{wt} \% \mathrm{PdS}$ prepared by hydrothermal 


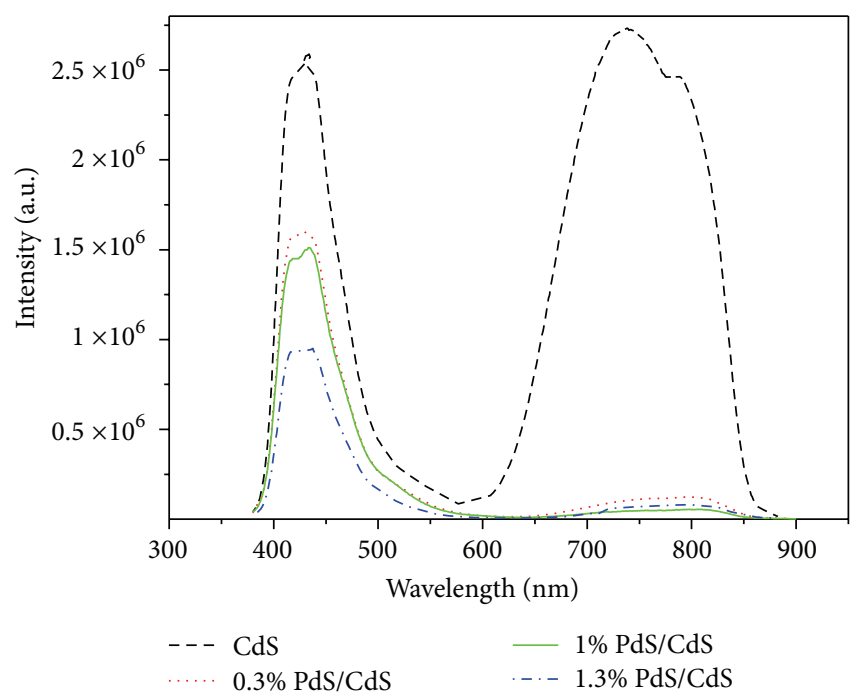

(a)

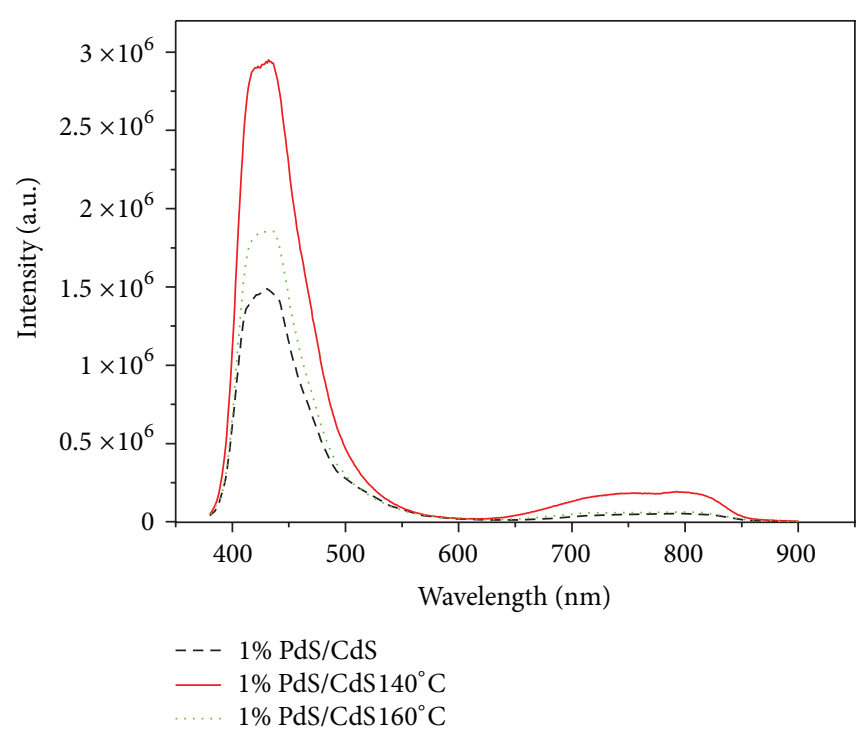

(b)

Figure 3: (a) Photoluminescence spectra of PdS/CdS samples loaded with different amounts of PdS. (b) Photoluminescence spectra of $\mathrm{PdS} / \mathrm{CdS}$ samples loaded with $1 \mathrm{wt} \% \mathrm{PdS}$ prepared by different methods.

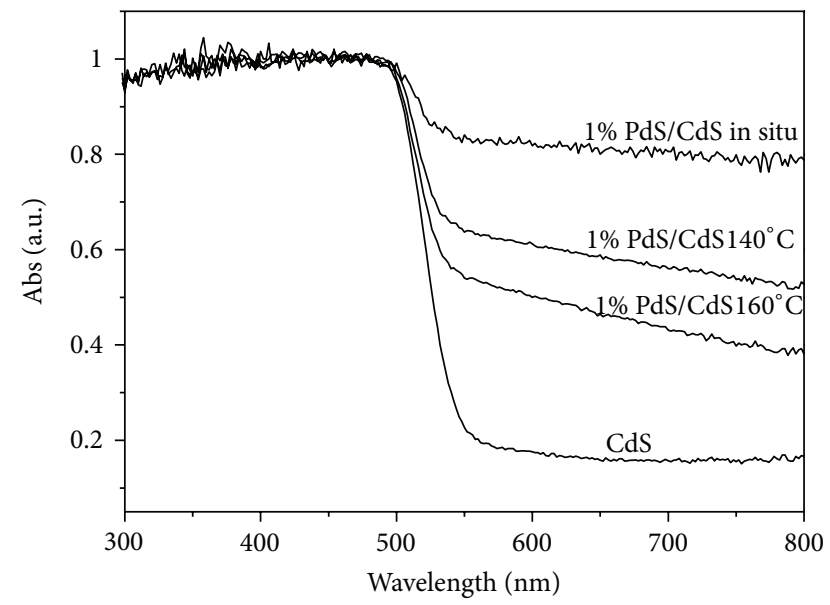

FIGURE 4: UV-visible absorption spectra of CdS and PdS/CdS samples.

method. It appears that the photocatalytic activity also decreases slightly. But the photocatalytic stability improves, as shown in Figure 2 which shows the hydrogen evolution on $\mathrm{PdS} / \mathrm{CdS}$ photocatalysts loaded with $1 \% \mathrm{PdS}$ prepared by different methods as long as $\mathrm{Na}_{2} \mathrm{~S}_{/} \mathrm{Na}_{2} \mathrm{SO}_{3}$ is present. $\mathrm{PdS} / \mathrm{CdS}$ photocatalysts prepared by the in situ coprecipitation can act stably for less than 40 hours. It is noted that $\mathrm{PdS} / \mathrm{CdS}$ photocatalysts prepared by hydrothermal method can exhibit significantly improved stability, showing steady hydrogen production more than 70 hours. The observation suggests that the dispersion of PdS in nanostructured CdS can improve the photoactivity, but the stable photocatalytic activity for hydrogen evolution can be influenced by the synthesis method.

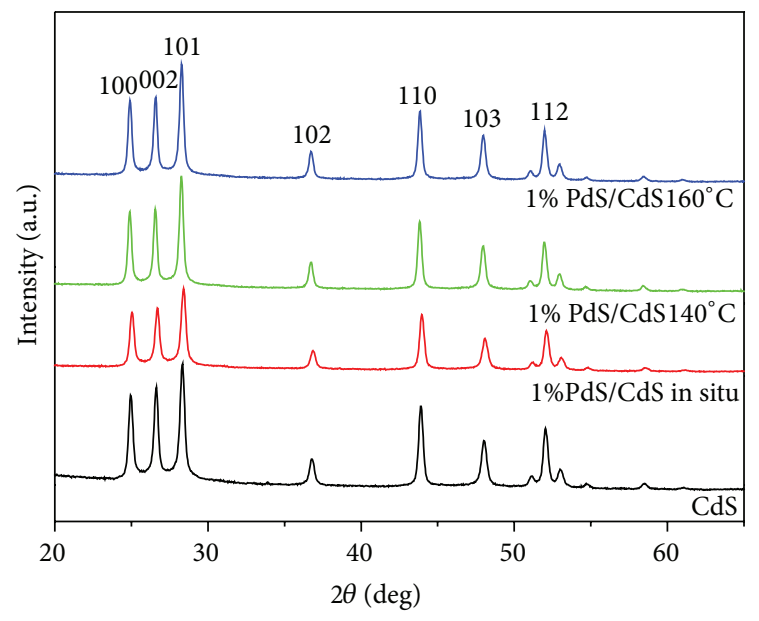

FIGURE 5: XRD patterns of PdS/CdS samples.

To analyze the reason, Figure 3 shows the photoluminescence spectra of $\mathrm{PdS} / \mathrm{CdS}$ photocatalysts. The photoluminescence spectroscopy can give information about the photoexcited energy/electron transfer and recombination process, and it has been widely used to investigate the photophysical and photochemical properties of photocatalysts [23]. As shown in Figure 3(a), the pure CdS exhibits broad fluorescence peak related to Stoke's shifts $\sim 435 \mathrm{~nm}$ and $\sim 750 \mathrm{~nm}$ [4]. The band at $\sim 432 \mathrm{~nm}$ is due to intrinsic emission, whereas that at $750 \mathrm{~nm}$ originates from transition of electrons rapped at surface state to the valance band of CdS. This fact suggests that nanostructured CdS has defects. By loading PdS, the fluorescence intensities are quenched, which reveals that the recombination of photoelectrons and holes is efficiently suppressed resulting in the improved photoactivity. 


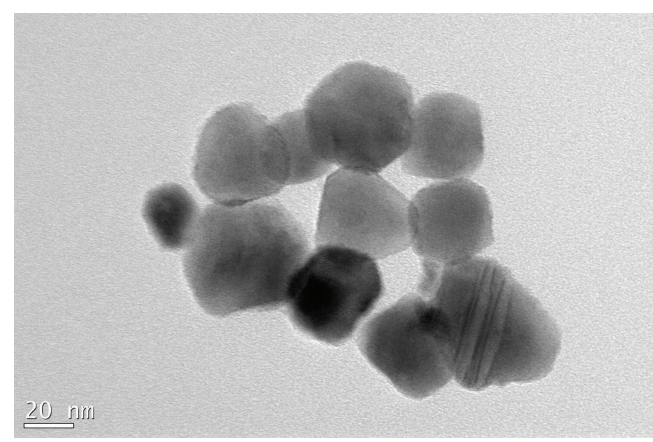

(a)

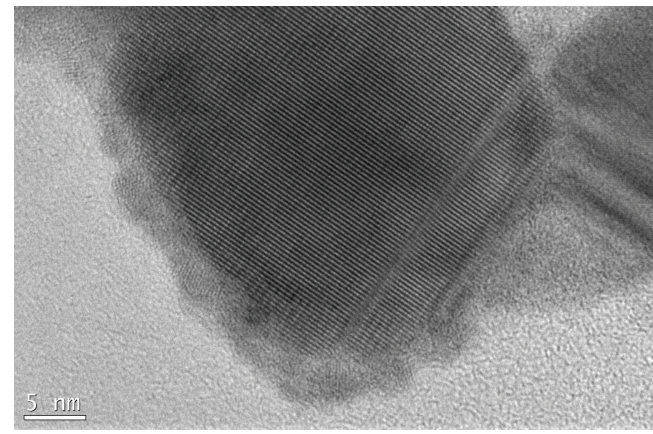

(c)

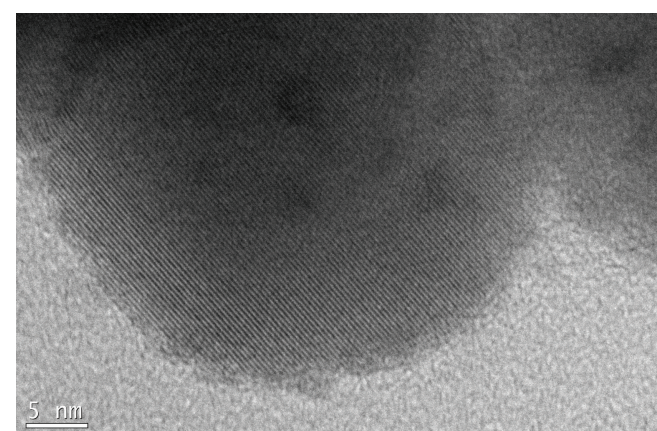

(b)

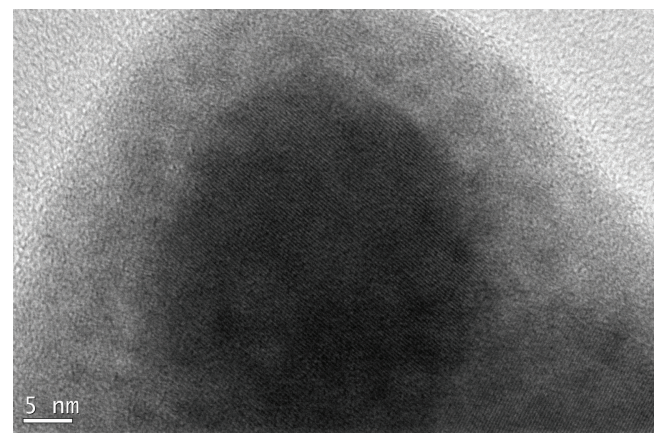

(d)

FIGURE 6: TEM image of ( $\mathrm{a}$ and b) CdS, (c) PdS/CdS prepared by in situ coprecipitation, and (d) PdS/CdS prepared by hydrothermal method at $160^{\circ} \mathrm{C}$.

However, the fluorescence intensities of PdS/CdS prepared by hydrothermal method are slightly enhanced as shown in Figure 3(b). This fact might be beneficial for the stability of photocatalysts. Then it can be concluded that the weaker the fluorescence intensity of the photocatalyst is, the higher the photoactivity is.

The UV-visible absorption spectra for PdS/CdS are shown in Figure 4. The pure $\mathrm{CdS}$ has an absorption edged at about $550 \mathrm{~nm}$, with energy gap estimated to be $2.3 \mathrm{eV}$. The spectra of PdS/CdS show an enhanced absorption in visible light region. Such an enhancement can be assigned to the existence of PdS phase. It was reported that PdS could not absorb visible light [4]. Therefore, the fact suggests that the PdS phase can enhance the absorption of CdS phase. From Figure 4, it can be seen that the enhancement of the absorption in visible light region is different which is dependent on the preparation condition. It suggests that the higher the visible adsorption is, the higher the photoactivity is.

The XRD patterns of various samples displayed in Figure 5 can be readily indexed as the (100), (002), (101), (110), (103), and (112) planes of the hexagonal phase of the wurtzite CdS structure, which are well matched with the standard values (JCPDS Card No. 02-0549) according to the main diffraction peaks at $24.9^{\circ}, 26.6^{\circ}, 28.4^{\circ}, 43.7^{\circ}, 48.1^{\circ}$, and $52.0^{\circ}$. It is worth noting that there are no characteristic peaks associated with PdS in these XRD patterns, which may be due to the low concentration of PdS and its relatively low crystallinity for the $\mathrm{PdS} / \mathrm{CdS}$ samples prepared by the in situ coprecipitation and due to the high dispersion of PdS particles in the nanostructured CdS and the low concentration of PdS for the PdS/CdS samples prepared by hydrothermal method. By the way the diffraction peak would not shift when $\mathrm{Pd}^{2+}$ was doped into the lattice of $\mathrm{CdS}$, considering that an ionic radius of $\mathrm{Pd}^{2+}(0.88 \AA)$ is lower than that of $\mathrm{Cd}^{2+}(0.97 \AA)$. The fact indicates that no significant lattice deformation occurred with different treatments of PdS/CdS samples.

Morphology of the as-prepared photocatalysts is shown in Figure 6. From Figure 6(a), it can be seen that the nanostructured CdS is mainly composed of nanoshperes. As shown in Figure 6(b), for the higher magnification of $\mathrm{CdS}$, these nanospheres are well crystallized. When PdS is loaded, there appears spherical nanoparticles on the surface of CdS which disperse more uniformly by comparing Figures 6(b) and 6(c). PdS by itself displays no photoactivity for $\mathrm{H}_{2}$ production, but $\mathrm{PdS}$ can act as a cocatalyst together with CdS. Then it suggests that PdS exists on the surface of $\mathrm{CdS}$ which can enhance the photoactivity of CdS. However, there appears a nanoparticle layer for $\mathrm{PdS} / \mathrm{CdS}$ prepared by hydrothermal method as shown in Figure 6(d). This indictes that the loading of PdS can improve the photoactivity of CdS, but the nanoparticle layer may decrease the 
photoactivity in spite of the increase in the stability of CdS.

\section{Conclusion}

Nanostructured PdS/CdS photocatalysts were synthesized by an in situ coprecipitation and a hydrothermal method, respectively. With a concentration of $\mathrm{PdS}$ approximately $1.0 \%, \mathrm{PdS} / \mathrm{CdS}$ photocatalysts synthesized by the in situ coprecipitation displayed the highest photocatalytic activity for hydrogen evolution under visible light irradiation from an aqueous solution containing sulfide and sulfite. However, PdS/CdS photocatalysts synthesized by the hydrothermal method showed the highest stability for hydrogen evolution. This study indicates that it is an effective method for preparing a stable, efficient photocatalyst by using a suitable cocatalyst.

\section{Acknowledgments}

This work was financially supported by the National Basic Research Program of China (2009CB220000), Research Fund for the Doctoral Program of Higher Education of China (20110201120042), and Natural Science Foundation of China (21206133).

\section{References}

[1] X. B. Chen, S. H. Shen, L. J. Guo, and S. S. Mao, "Semiconductorbased photocatalytic hydrogen generation," Chemical Reviews, vol. 11, pp. 6503-6570, 2010.

[2] C. L. Li, J. Yuan, B. Y. Han, and W. F. Shanguan, "Synthesis and photochemical performance of morphology-controlled CdS photocatalysts for hydrogen evolution under visible light," International Journal of Hydrogen Energy, vol. 36, no. 7, pp. 4271-4279, 2011.

[3] S. H. Shen, P. H. Guo, J. W. Shi, and L. J. Guo, "Visible-lightdriven photocatalytic water splitting on nanostructured semiconducting materials," International Journal of Nanotechnology, vol. 8, no. 6-7, pp. 523-591, 2011.

[4] S. Zhang, Q. Y. Chen, D. W. Jing, Y. H. Wang, and L. J. Guo, "Visible photoactivity and antiphotocorrosion performance of PdS-CdS photocatalysts modified by polyaniline," International Journal of Hydrogen Energy, vol. 37, pp. 791-796, 2012.

[5] A. Fujishima and K. Honda, "Electrochemical photolysis of water at a semiconductor electrode," Nature, vol. 238, no. 5358, pp. 37-38, 1972.

[6] E. Borgarello, N. Serpone, E. Pelizzetti, and M. Barbeni, "Efficient photochemical conversion of aqueous sulphides and sulphites to hydrogen using a rhodium-loaded CdS photocatalyst," Journal of Photochemistry, vol. 33, no. 1, pp. 35-48, 1986.

[7] I. Tsuji and A. Kudo, " $\mathrm{H}_{2}$ evolution from aqueous sulfite under visible-light irradiation over $\mathrm{Pb}$ and halogen-codoped $\mathrm{ZnS}$ photocatalysts," Journal of Photochemistry and Photobiology A, vol. 156, no. 1-3, pp. 249-252, 2003.

[8] M. Matsumura, S. Furukawa, Y. Saho, and H. Tsubomura, "Cadmium sulfide photocatalyzed hydrogen production from aqueous solutions of sulfite: effect of crystal structure and preparation method of the catalyst," Journal of Physical Chemistry, vol. 89, no. 8, pp. 1327-1329, 1985.
[9] K. G. Kanade, B. Jin-OoK, D. P. Amalnerkar, and B. B. Kale, "Nano-CdS by polymer-inorganic solid-state reaction: visible light pristine photocatalyst for hydrogen generation," Materials Research Bulletin, vol. 41, no. 12, pp. 2219-2225, 2006.

[10] H. Zhang and Y. F. Zhu, "Significant visible photoactivity and antiphotocorrosion performance of CdS photocatalysts after monolayer polyaniline hybridization," Journal of Physical Chemistry C, vol. 114, no. 13, pp. 5822-5826, 2010.

[11] J. S. Jang, S. M. Ji, S. W. Bae, H. C. Son, and J. S. Lee, "Optimization of $\mathrm{CdS} / \mathrm{TiO}_{2}$ nano-bulk composite photocatalysts for hydrogen production from $\mathrm{Na}_{2} \mathrm{~S}_{2} \mathrm{Na}_{2} \mathrm{SO}_{3}$ aqueous electrolyte solution under visible light $(\lambda \geq 420 \mathrm{~nm})$," Journal of Photochemistry and Photobiology A, vol. 188, no. 1, pp. 112-119, 2007.

[12] A. Eychmuller, "Structure and photophysics of semiconductor nanocrystals," The Journal of Physical Chemistry B, vol. 104, pp. 6514-6528, 2000.

[13] J. S. Jang, D. J. Ham, N. Lakshminarasimhan, W. Y. Choi, and J. S. Lee, "Role of platinum-like tungsten carbide as cocatalyst of CdS photocatalyst for hydrogen production under visible light irradiation," Applied Catalysis A, vol. 346, no. 1-2, pp. 149-154, 2008.

[14] I. B. Rufus, V. Ramakrishnan, B. Viswanathan, and J. C. Kuriacose, "Photoelctrochemical and photocatalytic studies on Pd/CdS," Indian Journal of Technology, vol. 27, pp. 171-173, 1989.

[15] J. B. Zhong, Y. Lu, W. D. Jiang et al., "Characterization and photocatalytic property of $\mathrm{Pd} / \mathrm{TiO}_{2}$ with the oxidation of gaseous benzene," Journal of Hazardous Materials, vol. 168, no. 2-3, pp. 1632-1635, 2009.

[16] A. Khalil, M. A. Gondal, and M. A. Dastageer, "Augmented photocatalytic activity of palladium incorporated $\mathrm{ZnO}$ nanoparticles in the disinfection of Escherichia coli microorganism from water," Applied Catalysis A, vol. 402, no. 1-2, pp. 162-167, 2011.

[17] B. Neppolian, A. Bruno, C. L. Bianchi, and M. Ashokkumar, "Graphene oxide based $\mathrm{Pt}-\mathrm{TiO}_{2}$ photocatalyst: ultrasound assisted synthesis, characterization and catalytic efficiency," Ultrasonics Sonochemistry, vol. 19, pp. 9-15, 2011.

[18] N. Sahu, S. N. Upadhyay, and A. S. K. Sinha, "Kinetics of reduction of water to hydrogen by visible light on alumina supported Pt-CdS photocatalysts," International Journal of Hydrogen Energy, vol. 34, no. 1, pp. 130-137, 2009.

[19] J. G. Yu, W. G. Wang, and B. Cheng, "Synthesis and enhanced photocatalytic activity of a hierarchical porous flowerlike p-n junction $\mathrm{NiO} / \mathrm{TiO}_{2}$ photocatalyst," Chemistry, vol. 5, no. 12, pp. 2499-2506, 2010.

[20] A. A. Ismail, L. Robben, and D. W. Bahnemann, "Study of the efficiency of UV and visible-light photocatalytic oxidation of methanol on mesoporous $\mathrm{RuO}_{2}-\mathrm{TiO}_{2}$ nanocomposites," ChemPhysChem, vol. 12, no. 5, pp. 982-991, 2011.

[21] X. Zong, H. J. Yan, G. P. Wu et al., "Enhancement of photocatalytic $\mathrm{H}_{2}$ evolution on CdS by loading $\mathrm{MoS}_{2}$ as cocatalyst under visible light irradiation," Journal of the American Chemical Society, vol. 130, no. 23, pp. 7176-7177, 2008.

[22] H. J. Yan, J. H. Yang, G. J. Ma et al., "Visible-light-driven hydrogen production with extremely high quantum efficiency on Pt-PdS/CdS photocatalyst," Journal of Catalysis, vol. 266, no. 2, pp. 165-168, 2009.

[23] H. P. Liu, K. Zhang, D. W. Jing, G. J. Liu, and L. J. Guo, "SrS/CdS composite powder as a novel photocatalyst for hydrogen production under visible light irradiation," International Journal of Hydrogen Energy, vol. 35, no. 13, pp. 7080-7086, 2010. 

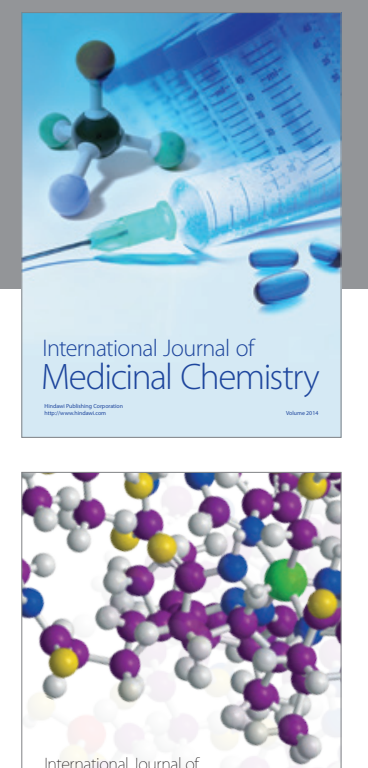

\section{Carbohydrate} Chemistry

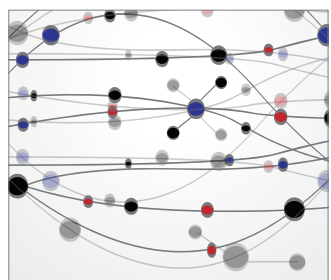

The Scientific World Journal
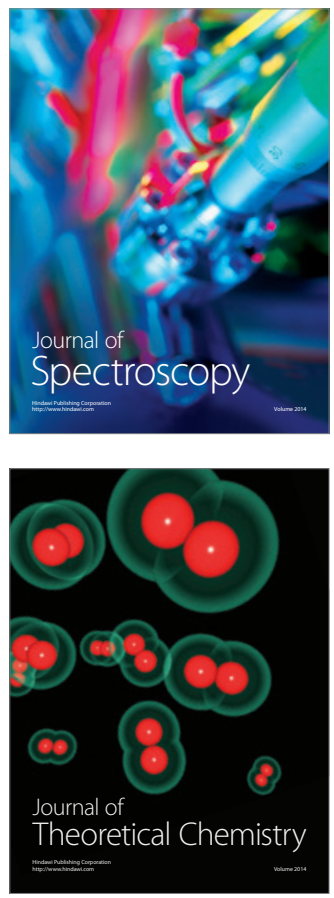
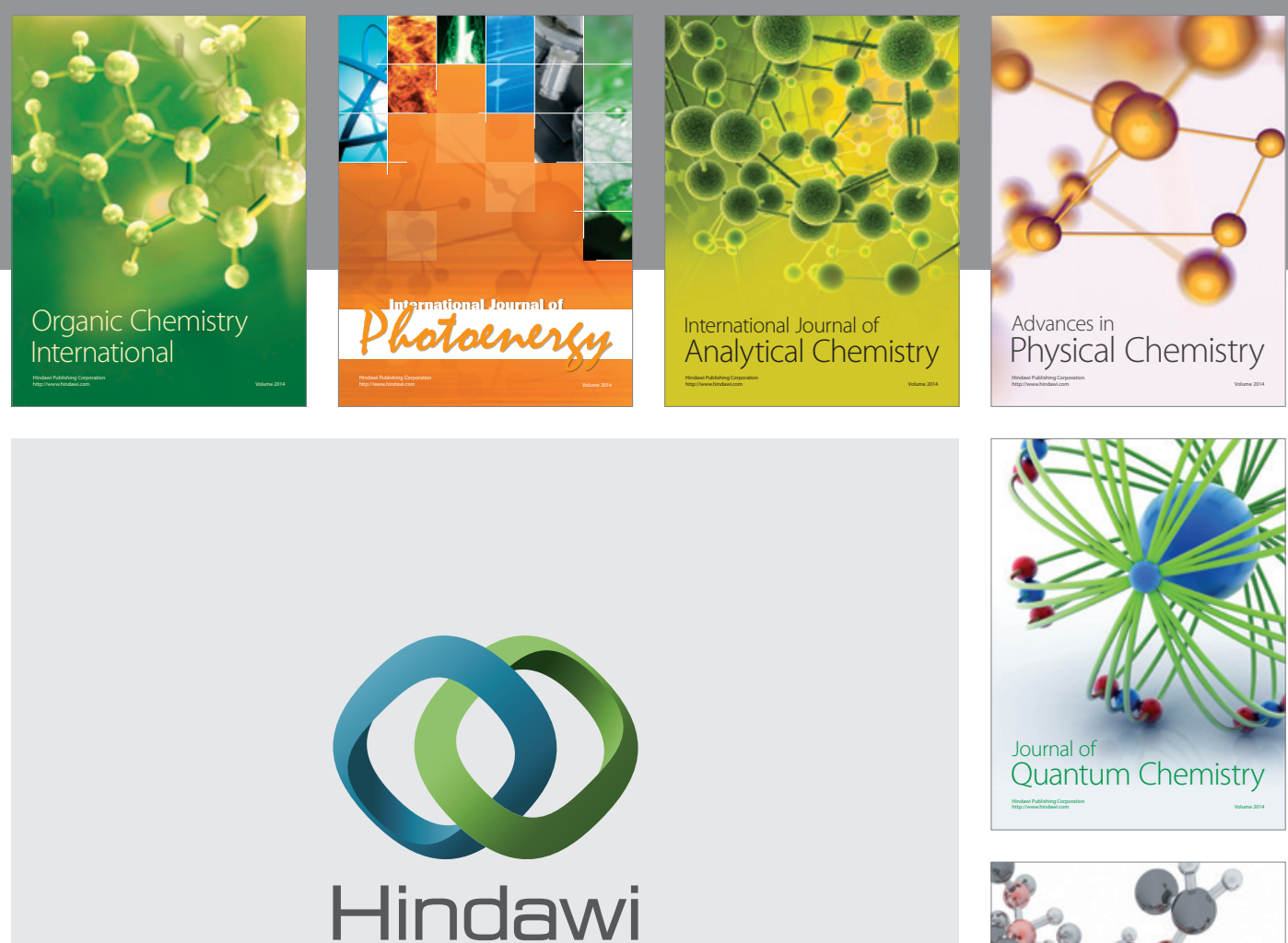

Submit your manuscripts at

http://www.hindawi.com

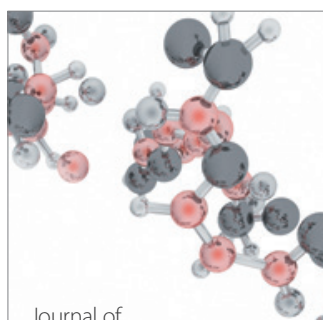

Analytical Methods

in Chemistry

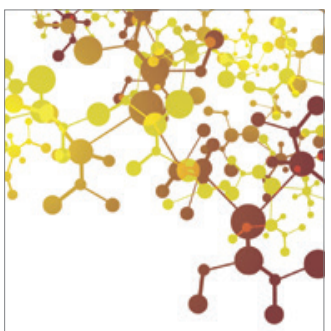

Journal of

Applied Chemistry

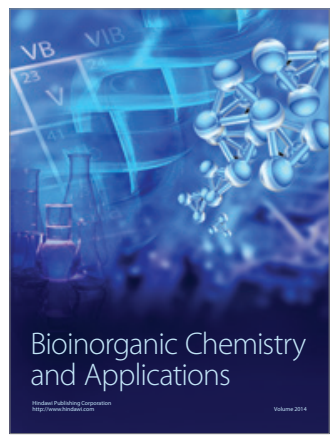

Inorganic Chemistry
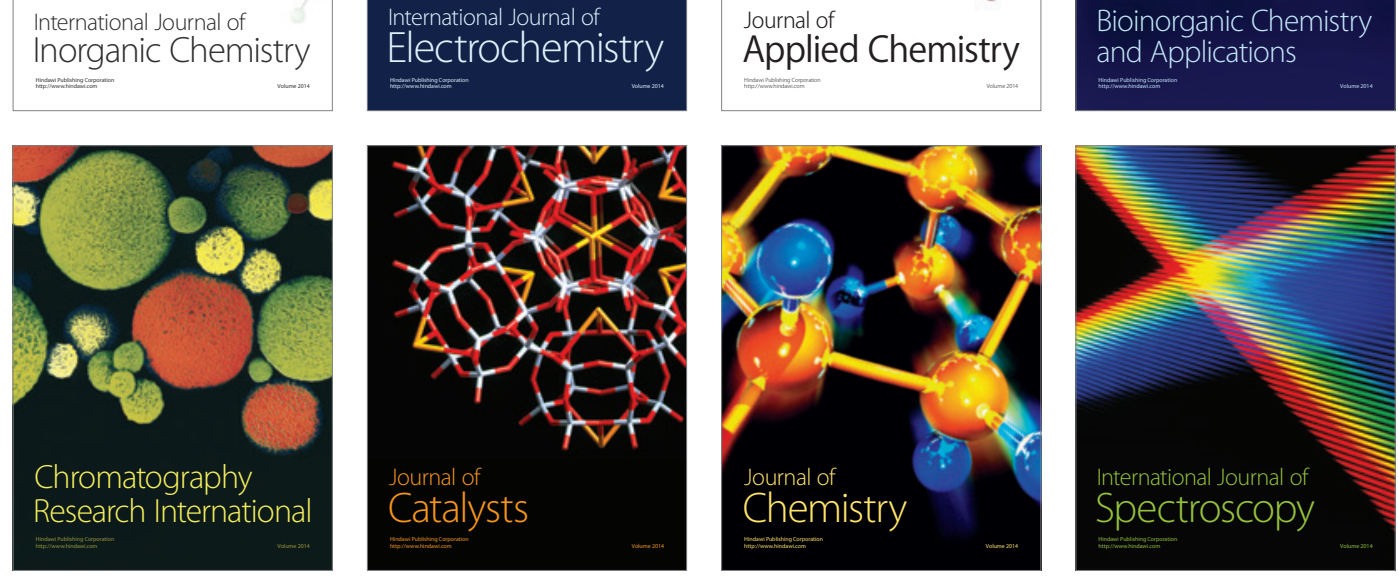